\title{
Correspondence
}

\section{Is there an association between season of birth and blood DNA methylation in adulthood?}

Pierre-Antoine Dugué ${ }^{1,2}$, Yvonne M Geurts ${ }^{1}$, Roger L Milne ${ }^{1,2}$

${ }^{1}$ Cancer Epidemiology Centre, Cancer Council Victoria, Melbourne, VIC 3004, Australia

${ }^{2}$ Centre for Epidemiology and Biostatistics, Melbourne School of Population and Global

Health, University of Melbourne, Parkville, VIC 3052, Australia

\section{Dear Editor,}

In a recent article published in your journal (1), Lockett et al. concluded that DNA methylation in adulthood is associated with season of birth and suggested that this association arises post-natally and could mediate the effect of season of birth on allergy. Their epigenome-wide association study was based on methylation measured in whole-blood DNA from 367 participants aged 18 from the United Kingdom. The authors reported that $92 \mathrm{CpG}$ sites were associated with specific seasons of birth and that four of these associations were replicated in an independent series of 207 children aged eight from the Netherlands.

We attempted to replicate these 92 associations using 2,774 adults aged 40 to 70 who were selected as controls in one of six case-control studies nested in the Melbourne Collaborative Cohort Study (2). Season of birth was defined as in Lockett et al. for individuals born in the Northern Hemisphere (UK, N=186; Italy, Greece, and Malta, N=712), and reversed for people born in the Southern Hemisphere (Australia and New-Zealand, N=1,876). We used linear mixed effects models for methylation M-values (measured using the same HM450K assay) including as fixed effects the variables used in Lockett et al. (except maternal socioeconomic status), as well as age at blood draw, country of birth, and sample type (peripheral blood mononuclear cells, dried blood spots, buffy coats), and as random effects the study in which the sample was processed and the plate and chip of the assay (3). Results for four of the $92 \mathrm{CpG}$ sites were consistent in direction with those of Lockett et al. and had a p-value less than 0.05 (Table 1), but none was significant after Bonferroni correction for This is the author manuscript accepted for publication and has undergone full peer review but has not been through the copyediting, typesetting, pagination and proofreading process, which may lead to differences between this version and the Version of Record. Please cite this article as doi: $10.1111 /$ all.12949

This article is protected by copyright. All rights reserved 
multiple testing. Neither the four signals that Lockett et al. reported as replicated in the Dutch study nor the strongest hit from their discovery phase (cg07175945) were nominally significant in our data $(\mathrm{P}>0.05)$. Our results were consistent in sensitivity analyses which included: further adjusting for blood cell composition derived from the Houseman algorithm; further adjusting for smoking, BMI and alcohol drinking; and stratifying the analyses by region of birth (Australia/NZ, UK, Southern Europe). We did not find clear evidence of season-associated differences in methylation at other CpGs on the HM450K assay $\left(\mathrm{P}>10^{-7}\right)$.

A possible explanation for the general lack of replication of the Lockett et al. findings in our study is the older and wide-ranging age of our participants. However, the $92 \mathrm{CpGs}$ assessed were not strongly associated with age (data not shown), and for most the mean methylation level was similar to that of Lockett et al. (Table 1), suggesting that methylation at these CpGs remains relatively stable throughout adulthood. Another limitation of our study is heterogeneity in the birth place of participants, although similarly null results were observed in stratified analyses by region of birth, in particular for UK-born participants only.

Consistent with our results is the observation that none of the four nominally significant associations presented in Lockett et al.'s replication study was significant after Bonferroni or FDR correction for multiple testing. Taken together, these findings suggest that the vast majority of the 92 CpGs identified as associated with season of birth using the 'ttScreening' method may be false-positives. This general lack of replication of associations identified using this method has also been observed for maternal smoking $(4,5)$.

In our view, there is currently limited evidence that season of birth influences blood DNA methylation in adulthood. More research is needed, both to more definitively identify associations and to demonstrate that these mediate the effect of season of birth on allergy.

Words: 591

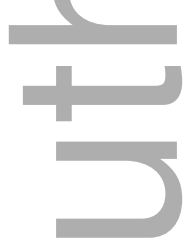

\section{References}


1. Lockett GA, Soto-Ramirez N, Ray MA, Everson TM, Xu CJ, Patil VK, et al. Association of Season of Birth with DNA Methylation and Allergic Disease. Allergy 2016; DOI:

10.1111/all.12882.

2. Giles GG, English DR. The Melbourne Collaborative Cohort Study. IARC Sci Publ 2002;156:69-70.

3. Dick KJ, Nelson CP, Tsaprouni L, Sandling JK, Aissi D, Wahl S, et al. DNA methylation and body-mass index: a genome-wide analysis. Lancet 2014;383:1990-1998.

4. Ray MA, Tong X, Lockett GA, Zhang H, Karmaus WJ. An Efficient Approach to Screening Epigenome-Wide Data. Biomed Res Int 2016;2016:article ID 2615348.

5. Joubert BR, Felix JF, Yousefi P, Bakulski KM, Just AC, Breton C, et al. DNA Methylation in Newborns and Maternal Smoking in Pregnancy: Genome-wide Consortium Meta-analysis. Am J Hum Genet 2016;98(4):680-696.

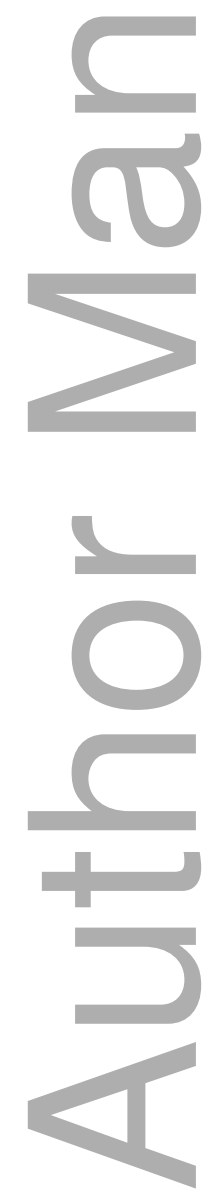




\section{Correspondence}

\section{Epigenetic association with season of birth: a complex relationship}

Gabrielle A. Lockett ${ }^{1}$, Hongmei Zhang ${ }^{2}$, Wilfried Karmaus ${ }^{2}$, John W. Holloway ${ }^{1,3}$

${ }^{1}$ Human Development and Health, Faculty of Medicine, University of Southampton, Southampton, UK

${ }^{2}$ Division of Epidemiology, Biostatistics and Environmental Health, School of Public Health, University of Memphis, Memphis, TN, USA

${ }^{3}$ Clinical and Experimental Sciences, Faculty of Medicine, University of Southampton, Southampton, UK

\section{Dear Editor,}

We recently reported the discovery of season of birth-associated DNA methylation, constituting a plausible molecular mechanism for the effect of season of birth on long-lasting outcomes such as allergic disease, for the first time (1). We write in response to the analysis of season of birthassociated DNA methylation by Dugué and colleagues, from which they conclude the set of season of birth-associated $\mathrm{CpGs}$ that we identified may include false positives. We value the contribution of Dugue et al. to the investigation of the hypothesis that epigenetic effects mediate the observed relationship between season of birth and allergic disease. However, we note there are differences between our analytical approaches that may account for the differences observed between the two studies.

One significant issue is the choice of confounders to include in analytical models. This is of crucial importance for epigenetic analyses that can be confounded by both experimental (e.g. chip, batch effects) and unaccounted environmental effects. For example, Dugué et al. made no adjustment for maternal socioeconomic status (SES). While it may initially seem surprising, there is strong evidence that SES causally influences a person's season of birth, with high SES mothers giving birth more often in spring and less often in winter, resulting in annual cyclical variation in SES (2). Because SES is also associated with DNA methylation in adulthood (3), this variable may confound an association of season of birth with DNA methylation, and therefore must be adjusted for. Interestingly, the CpG site with the lowest $p$ value in Dugué et al.'s validation, cg00787537, was also the most statistically significant $\mathrm{CpG}$ in our preliminary analyses with models omitting 
SES. This could indicate that their attempted validation of our sites was primarily hindered by their different statistical model, especially failing to adjust for maternal SES.

Secondly, there are other differences between the cohorts in question. As acknowledged by Dugué et al., there is a difference in the mean age of participants in the two studies. Birth seasonassociated methylation could be present up to age 18 and fade before age 40-70, without necessarily being significantly age-dependent.

One issue that Dugué et al. highlighted as an explanation for the non-replication of our observations in their study was our choice of analytical approach, namely Training-Testing (TT) as implemented in the R-package ttScreening. However, we have shown that epigenome-wide association studies (EWASs) using the TT method actually do replicate results obtained using standard statistical methods; in fact TT may be more sensitive than standard single-sample-set analysis methods (4). A direct comparison found overlapping sets of CpGs were identified by TT, FDR and Bonferroni methods (4). Of the CpGs TT identified in adult blood DNA associated with exposure to maternal smoking in utero, $31 \%$ of $\mathrm{CpG}$ and $63 \%$ of genes were subsequently confirmed in a meta-analysis of cord blood DNA (5), suggesting that they are indeed true positives. In addition the ttScreening approach corrects for surrogate variables: by identifying and adjusting for these in the EWAS model, unknown structure in the data can be taken in to account. We therefore recommend TT-based methods such as ttScreening to other groups performing EWASs.

In summary, while the association between season of birth and a range of diseases has long been observed, the mechanisms underlying these observations remain uncertain. While we presented data suggesting epigenetic programming may be one mechanism accounting for these effects, Dugue et al.'s study highlights the need for further large-scale meta-analyses of epigenetic data to confirm this. Consortia such as the Pregnancy and Childhood Epigenetics (PACE) consortium (http://www.niehs.nih.gov/research/atniehs/labs/epi/pi/genetics/pace/index.cfm) will be essential to this process, allowing for meta-analyses and shared analytical approaches.

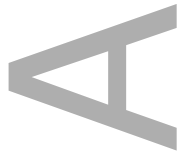

Word count: 599 
1. Lockett GA, Soto-Ramírez N, Ray MA, Everson TM, Xu C-J, Patil VK, et al. Association of season of birth with DNA methylation and allergic disease. Allergy. Accepted.

2. Buckles KS, Hungerman DM. Season of birth and later outcomes: Old questions, new answers. Rev Econ Stat. 2012;95(3):711-24.

3. Borghol N, Suderman M, McArdle W, Racine A, Hallett M, Pembrey M, et al. Associations with early-life socio-economic position in adult DNA methylation. Int $J$ Epidemiol. 2012;41(1):6274.

4. Ray MA, Tong X, Lockett GA, Zhang H, Karmaus WJJ. An efficient approach to screening epigenome-wide data. BioMed Research International. 2016;2016:article ID 2615348.

5. Joubert BR, Felix JF, Yousefi P, Bakulski KM, Just AC, Breton C, et al. DNA methylation in newborns and maternal smoking in pregnancy: Genome-wide consortium meta-analysis. The American Journal of Human Genetics. 2016;98(4):680-96.

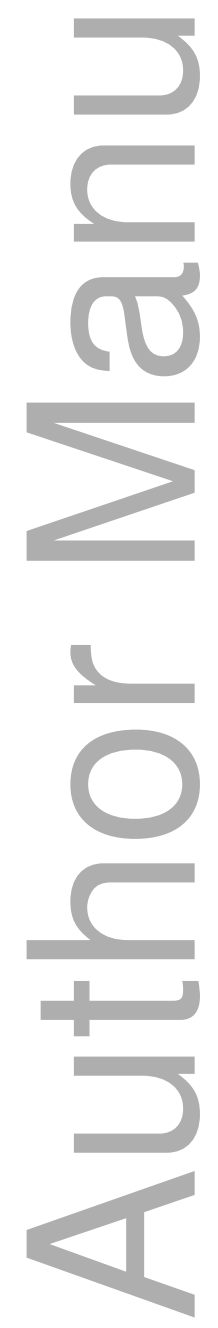




\section{University Library}

\section{- M M N E R VA A gateway to Melbourne's research publications}

Minerva Access is the Institutional Repository of The University of Melbourne

Author/s:

Dugue, P-A;Geurts, YM;Milne, RL

Title:

Is there an association between season of birth and blood DNA methylation in adulthood?

Date:

2016-10-01

Citation:

Dugue, P. -A., Geurts, Y. M. \& Milne, R. L. (2016). Is there an association between season of birth and blood DNA methylation in adulthood?. ALLERGY, 71 (10), pp.1501-1503. https:// doi.org/10.1111/all.12949.

Persistent Link:

http://hdl.handle.net/11343/291724 